\title{
Delivering the future
}

\section{Drugs to treat diseases from cancer to AIDS could soon rely on short strands of RNA for their effects. But scientists must first work out how to navigate these fragments around the body. Nathan Blow reports.}

The remarkable ability of short sequences of synthetic RNA to interfere with messenger RNA and thereby silence the activity of specific genes has proved incredibly helpful to geneticists wrestling with genetic function. And the push to harness this RNA interference (RNAi) for therapeutic use is now beginning to make headway. In the six years since the first paper reporting RNAi gene silencing in mammals was published ${ }^{1}$, at least six therapeutic programmes based on the concept have moved into clinical trials.

"Progress in the field of RNAi therapeutics has occurred remarkably fast," says John Maraganore, president and chief executive of Alnylam Pharmaceuticals in Cambridge, Massachusetts. But delivering the sequences remains a problem. Initial clinical trials relied on 'local delivery', directly introducing short interfering RNAs (siRNAs) into the specific tissue they were to treat. But for true therapeutic value, the siRNAs need to be introduced systemically.

"Systemic delivery is the major issue right now," says Alan Sachs, vice-president for RNA therapeutics based at Sirna Therapeutics,

a wholly owned subsidiary of Merck in San Francisco.

Getting a small RNA to interfere with the right messenger RNA in the correct tissue and cell type at a safe, therapeutic level by systemic administration requires an exquisite degree of control - creating the need for different delivery vehicles and potentially even specialized targeting strategies. Animal studies ${ }^{2}$ have shown that it is possible for siRNAs delivered systemically to silence target genes. "What we have learned over the past couple of years is that systemic delivery

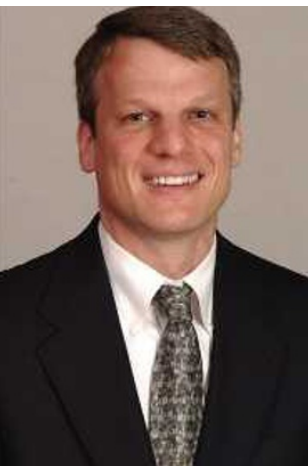

Alan Sachs says that delivery is the major issue for RNAi therapeutics. of RNAi can be achieved, and there are a variety of methods that can be used to achieve it," says Maraganore. But he is also quick to note that there is no simple solution.

"If you inject naked siRNA into the blood, under normal pressure, it doesn't work," says Daniel Anderson of the Center for Cancer Research at the Massachusetts Institute of Technology (MIT) in Cambridge. Yet naked high local concentrations of the RNA to be achieved, says Maraganore, while also taking advantage of naturally occurring mechanisms, such as pinocytosis, for uptake into the target cells.

Work by researchers at the company has also helped shed light on systemic delivery. In a 2004 study $^{2}$, they affirmed for the first time the potential 'drug-like' properties of siRNAs when

\section{THE VEHICLE LABORATORY}

Many companies are realizing that the development of new delivery vehicles for therapeutics based on RNA interference (RNAi) will require collaborative efforts. "We view the delivery of small RNAs as one of the most important biomedical endeavours in modern biological science," says John Maraganore, chief executive of Alnylam Pharmaceuticals in Cambridge, Massachusetts. "And when you have such a broad-scale challenge and opportunity, you can't do everything internally - you have to work with the best groups outside as well."

This spring, Alnylam initiated a collaboration with Daniel Anderson and Robert Langer at the Massachusetts Institute of Technology (MIT) in Cambridge. Under the agreement, Alnylam will provide funding for ten postdocs at MIT to work on issues directly related to small-RNA delivery while having the exclusive option to any intellectual property that comes out of the effort.

Anderson says that at its core this is an academic programme, so the goal is to get postdocs to work on the delivery issue while being trained as future leaders in the field.

"One nice feature is that we have collaborations with a company that is a leader in this field, so

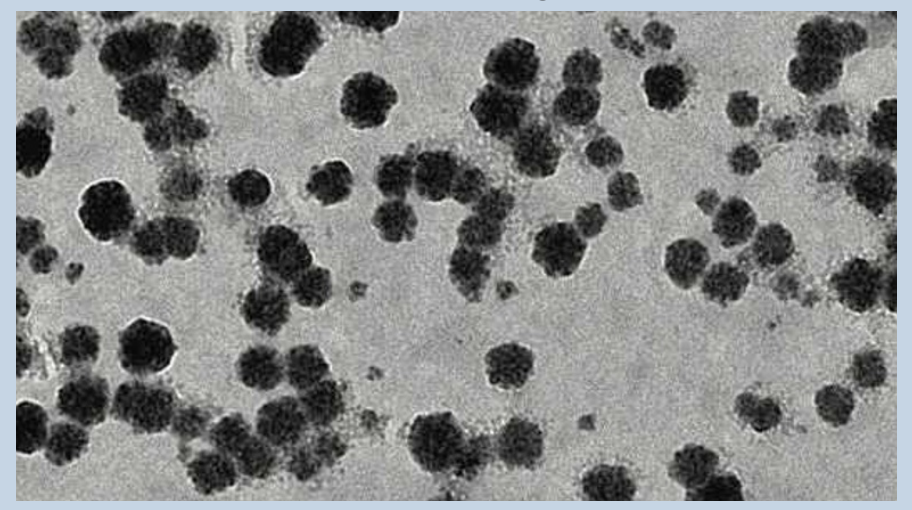

An electron microscope image of nanoparticles that can be used as

delivery vehicles for siRNA.

it allows us to accelerate our efforts tremendously," Anderson says. For example, when the MIT researchers develop any new delivery technologies, scientists at Alnylam can provide animal models and other methods to test and evaluate these new vehicles, providing quick feedback on whether or not the research is on the right track.
Langer's lab has a long history of translational research. It has developed principles that have led to some 40 products that are now in clinical trials or have been approved by the US Food and Drug Administration. "The hope is that we can do that here," says Langer. "We can do the kind of basic research to help solve the RNAi delivery problem and then work closely with Alnylam to take the basic research into the clinic where it can be used to treat different diseases."

In addition to the MIT collaboration, Alnylam also funds $R \& D$ and manufacturing activities in Vancouver, Canada, to further the development of cationic lipids for delivery, and has some 25 feasibility agreements in place in which Alnylam is testing and evaluating technology that has been introduced from either companies or academic groups. 
delivered systemically. The team used a synthetic RNA conjugated to cholesterol and stabilized with a partial phosphorothioate backbone and 2'-O-methyl sugar modifications on both the sense and antisense strands of the RNA.

Since this study, both lipid-and polymerbased vehicles for systemic delivery of siRNAs have been developed and tested. At Polyplus-transfection in Illkirch, France, researchers have taken advantage of the difference between cationic polymers and cationic lipids for systemic delivery to different organs. "We are interested in delivery to the lung and have used systemic administration of the cationic polymer polyethylenimine for delivery," says Patrick Erbacher, the company's chief scientific officer. "But for tumour injections, we use either a cationic polymer or a cationic lipid formulation."

With siRNAs conjugated to lipids or encapsulated in liposomes or lipid nanoparticles, several companies have achieved stable and efficient systemic delivery to organs including the liver, pancreas, kidneys and even to some types of tumour. And polymers that can com- plex with siRNA can deliver

the short sequences to organs such as the lungs, spleen and kidneys.

Researchers at Altogen Biosystems based in Las Vegas, Nevada, are exploring cationic lipids and biodegradable polymers for in vivo delivery, but have not found it easy. "There is no perfect method for delivery," says Andreas Kim, the company's vice-president of research and development.

"Nothing really works amazingly well. All methods have their advantages and disadvantages." In mice, he notes, lipid-based delivery of siRNA is very efficient but tends to induce an inflammatory response to the lipid formulation. Delivery vehicles based on biodegradable polymers, on the other hand, don't cause inflammatory responses but are not delivered as efficiently and the effects seem to be more transient than their lipidbased counterparts.
Alnylam is using liposomes to deliver siRNAs to the liver. "It is easier to target things in the liver with liposomes because about 95\% of the injected dose for liposomal formulations goes to the liver," Maraganore says. Liposomes are synthetic analogues of the cell membrane and are made up of hydrophilic and hydrophobic regions that form spherical 'packages' in aqueous conditions. Alnylam is using this approach to target two genes in the liver - one involved in regulating levels of low-density lipoprotein in the blood and the other involved in liver cancer (targeting both vascular endothelial growth factor and kinesin spindle protein).

The ability to use lipid-based delivery vehicles to target the liver has made the organ a popular starting point for many companies. Merck, for example, is using lipid nanoparti-

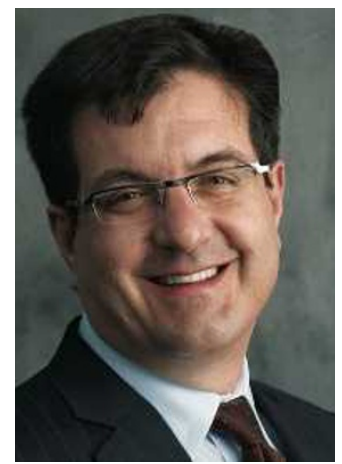

John Maraganore is confident systemic delivery of siRNA can be achieved. cles that, like liposomes, take advantage of endogenous mechanisms for uptake, but have no specific ligand attached for targeting.

Although lipid nanoparticles are its main focus, Merck is also exploring other delivery vehicles - in some cases through external collaborations. "We are working aggressively in the licensing arena - inviting people to work with us in an evaluation phase," says Sachs. In October, the company finalized a licensing agreement with

\section{THE INSIDE TRACK}

How to deliver short-interfering RNAs (siRNAs) to specific tissues is only part of the problem facing researchers. They also need to find out whether the RNA has reached its intended target. Anna Moore, a radiologist at Harvard Medical School in Boston, Massachusetts, is well aware of the issue. "There was no way to use a clinical imaging modality to see the delivery of siRNA," she says.

When researchers want to see whether an siRNA has been reached a particular tissue, they usually perform histological analysis followed by reverse transcription PCR to see whether the target gene was silenced. "You can do this with mice, but when you move on to humans it becomes impractical," Moore says.

Researchers can track siRNAs in vivo using bioluminescence imaging or by tracking green fluorescent proteins. But bioluminescence imaging is not a

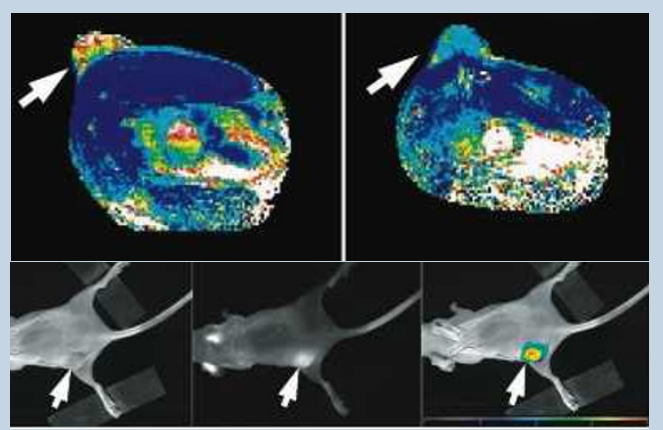

Now you see it: the nanoparticle system devised by Anna Moore's team allows siRNA delivery to be seen in MRI scans (top) and optical scans (bottom).

clinical modality. So Moore and her colleagues decided to try magnetic resonance imaging (MRI).

The first step was to design an siRNA delivery vehicle that could be imaged by MRI. Moore and her team used a nanoparticle containing an iron oxide core. They coated it with dextran, which could have various targeting features added to it relatively easily.
Although iron oxide can be imaged using MRI, the group also attached a fluorescent dye, Cy 5.5, to the dextran coat for optical imaging. "We wanted to correlate the imaging data with microscopic findings," says Moore.

The iron oxide nanoparticle generates a bright spot on the MRI image. The exact target of the nanoparticle can then be confirmed by the fluorescent dye and by doing microscopy for histological analysis.

Two further attachments were then made to the nanoparticle via the dextran coating: a membrane translocation peptide that can cross cell membranes and an
siRNA. With this, Moore and her colleagues thought they had a particle that could target and image delivery to tumour cells in vivo. But the imaging showed that the nanoparticle went to the liver and kidneys, and was present in other organs as well ${ }^{5}$.

Moore and her team plan to continue with the nanoparticles, trying to make them more efficient in terms of delivery and target uptake. But the real value of these nanoparticles might be their versatility. As different siRNAs or targeting peptides can be attached to the dextran coat, a large range of therapeutic siRNAs and peptides can be tested.

"My lab is really interested in imaging other pathologies such as diabetes, which is far from cancer but the imaging approaches are very similar," says Moore.

"And that is the beauty of this technology - you can apply it to different pathologies." 
Protiva Biotherapeutics of Burnaby, British Columbia in Canada, for its stable nucleic acid lipid particles (SNALPs). These are specialized lipid nanoparticles that encapsulate siRNA and were the first non-viral siRNA delivery vehicles that showed activity in non-human primates ${ }^{3}$. Alnylam has also launched a number of academic and industrial delivery collaborations (see 'The vehicle laboratory').

The rapid advancement of RNAi-based therapeutics is leading Polyplus-transfections to explore the manufacturing aspects of delivery vehicles. The company develops and markets DNA, RNA and protein transfection and delivery reagents for both in vitro and in vivo applications. And with RNAi therapeutics on the cusp of entering the clinic, the company sees the need to produce delivery vehicles in bulk under governmental quality specifications or 'current good manufacturing practices' (cGMP) standards. "If you want to be able to get these into the clinics, you have to have cGMP-qualified delivery systems," says Erbacher.

\section{Beyond the liver}

Despite this progress in general delivery, there is still some way to go for full therapeutic application. "I do not think that we are at the point now where we can name a tissue, specifically deliver and get good knockdown," says Anderson. Although he notes that there is good evidence to suggest that targeted systemic delivery should be possible.

Kim agrees that targeted delivery looks promising, but he cautions that the development of the delivery vehicles will be complicated. "You need a number of additional steps in the formulation process," he says.

A promising approach to targeted delivery involves incorporating targeting elements

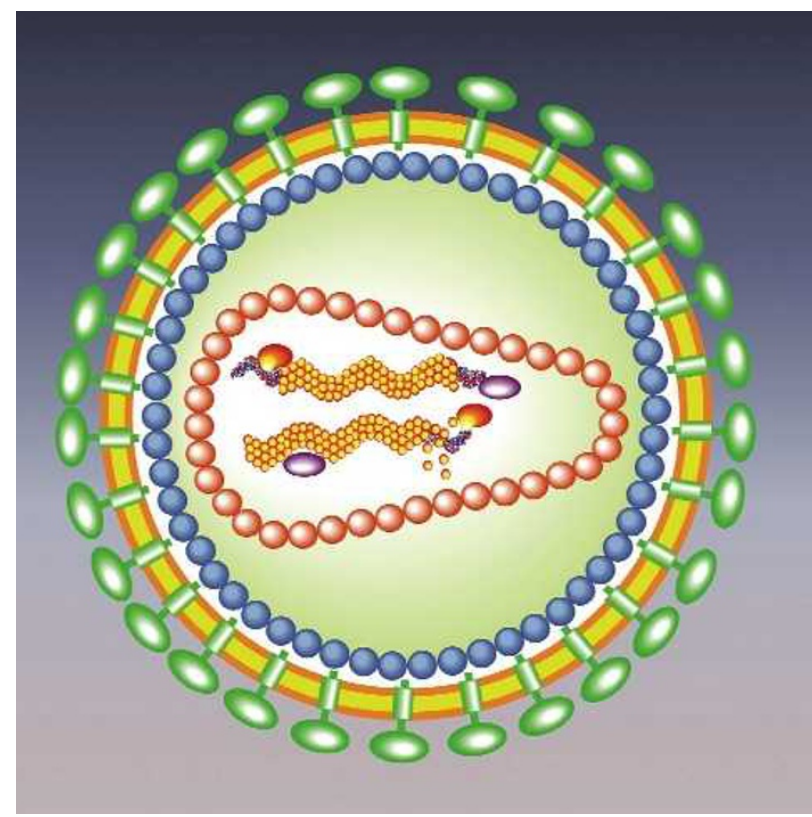

Lentiviruses, shown here schematically, can be modified to carry RNA into cells for potential therapeutic effect. such as antibody fragments, ligands and small chemical groups with the synthetic RNA. At the same time, techniques are being designed to image targeted nanoparticle delivery and biodistribution (see 'The inside track'). Some of the new approaches might overcome issues associated with lipid-based vehicles. For example, RNA aptamers attached to siRNAs, which have been used by researchers to target prostate cancer cells, might not cause the inflammatory responses seen in some cases with lipids or antibodies.

Another approach that might avoid inflammatory responses is being developed by Calando Pharmaceuticals in Pasadena, California, using polymers that contain cyclodextrin. When the polymers are mixed with siRNAs, they bind to the RNA backbone and assemble into nanoparticles. Targeting ligands or even stabilizing agents can then be attached to the cyclodextrin in the polymer to improve delivery.

\section{The evolutionary advantage}

Another approach to targeted delivery takes advantage of nature. "Viruses have learned how to get into cells - cross the membrane into the cytoplasm then get into the nucleus," says Inder Verma, a geneticist at the Salk Institute for Biological Studies in La Jolla, California. "All other approaches have to figure out how to do this." This gives viruses an "evo-

\section{THINKING SMALL}

"Each day you see major, significant publications on the roles of microRNAs in regulating pathways of genes involved in disease aetiology," says John Maraganore of Alnylam Pharmaceuticals in Cambridge, Massachusetts. Alongside the research into the mechanisms and roles of microRNA (miRNA) in disease, researchers are now starting to look at the potential of miRNA-based therapeutics.

Regulus Therapeutics in Carlsbad, California, is one of the first companies to be founded entirely for the development of miRNA-based therapeutics. It was formed in September as a joint venture between Alnylam and Isis Pharmaceuticals in Carlsbad. "We recognized that miRNAs were becoming a potential therapeutic opportunity," says Frank Bennett, senior vice-president of research at Isis, "but we also recognized that it was difficult for both companies to input the resources that were warranted." After much discussion, the two firms agreed to supply miRNA assets and core technologies to Regulus.

Regulus is taking two approaches to the development of miRNAbased therapeutics. "The most advanced approach is inhibiting the function of an endogenous miRNA in cells," says Bennett. This uses a synthetic oligonucleotide to

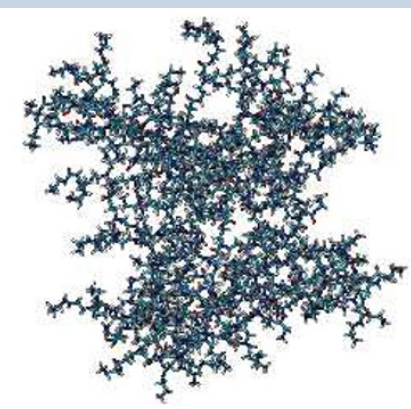

Testing of technologies, such as dendrimers, for delivering siRNAs may pave the way for miRNA drugs. target the miRNA for silencing. Although this is different from siRNA, as the miRNA is the target not the therapeutic agent, Bennett thinks that much of the technology developed for targeting mRNAs with siRNA is directly translatable to the targeting of miRNAs with oligonucleotides. And he notes that using oligonucleotides will also minimize any 'off-target' effects. "These oligonucleotides are very specific - even a single base mismatch will cause the oligo to lose activity," he says.

The second approach involves replacing miRNAs in or delivering them to cells, which Bennett says is similar to the current approach for siRNAs. The first applications here might be replacing miRNAs that are missing from diseaseassociated cells but present in a normal cells, which happens in some cancers, or augmenting naturally occurring miRNAs.

Although experience of siRNAs will help this nascent field, there is one unique issue in miRNA biology that researchers will have to address: miRNAs can inhibit tens to hundreds of genes at a time. Scores of researchers, both inside and outside academia, are now working to understand exactly how this regulation by miRNAs occurs. "You can use specific chemical modifications to the miRNA to limit that potential," says Bennett, "but it is still present."

Many researchers and companies now think miRNA-based therapeutics hold great promise for the future. And although some companies are waiting in the wings for more information on their basic mechanisms of action, others including Merck, Santaris Pharma of Hørsholm, Denmark, Rosetta Genomics in Rehovot, Israel, and Actigenics of MaurensScopont, France, are actively involved in miRNA research and development. 
lutionary advantage" as delivery vehicles for small RNAs, he adds.

By exploiting the natural diversity of viruses, researchers have developed a suite of viral vectors that can be used to deliver therapeutic sequences into a wide variety of cell types. Viral vectors have been around for some time and scientists are currently using them to deliver short hairpin RNA (shRNA) that can be processed by cells to yield siRNAs and silence genes.

Viral vectors from the DNA-based adenovirus and adeno-associated virus (AAV) have been used by researchers and companies, such as ArmaGen Technologies in Santa Monica, California, for shRNA delivery. AAV vectors have, however, found more widespread use in RNAi applications as these viruses can transduce both dividing and non-dividing cell types and result in stable, site-specific integration.

Lentivirus-based vectors are another option. "The utility of lentiviral vectors for RNAi research is their ability to transduce non-dividing cells and to efficiently transduce difficultto-transfect cells," says Boro Dropulic, founder and chief executive of Lentigen in Baltimore, Maryland.

But any viral vector will still have to overcome the issue that plagues synthetic RNAs: targeting. "Targeting is a big problem. There are several groups working on that issue now but the jury is still out," says Dropulic. What makes targeting viral vectors such a challenge is the difficulty in altering the viral structure with targeting elements while maintaining the proper function of viral particles.

For adenovirus and AAV vectors, for example, the viruses require a specific number of proteins to make their viral shell. Adding more proteins, such as an antibody fragment for targeting purposes, could be problematic. "Imagine the viral shell is like the dome of a sports centre," says Verma. "You can only put so much information in there, after which the dome will break." To overcome this problem, Verma thinks that researchers will need to find a way to modify the entire shell of the virus.

Lentiviral vectors are less problematic - the virus particles are surrounded by a lipid envelope in which proteins can be inserted without

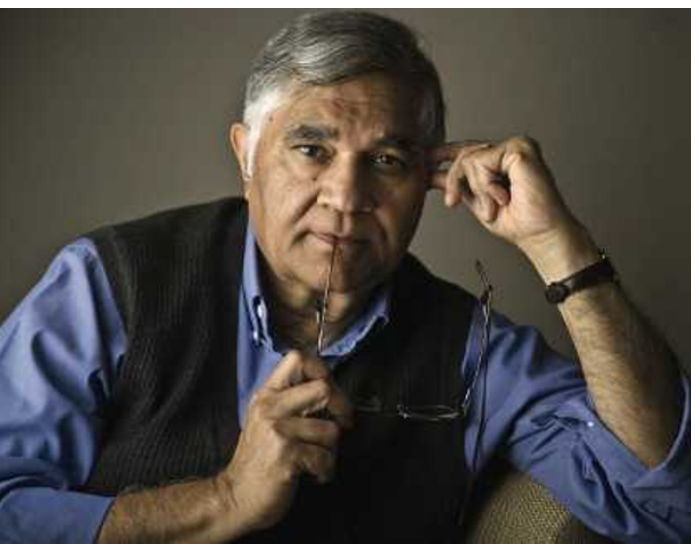

Inder Verma believes viruses have an evolutionary advantage for RNAi delivery.

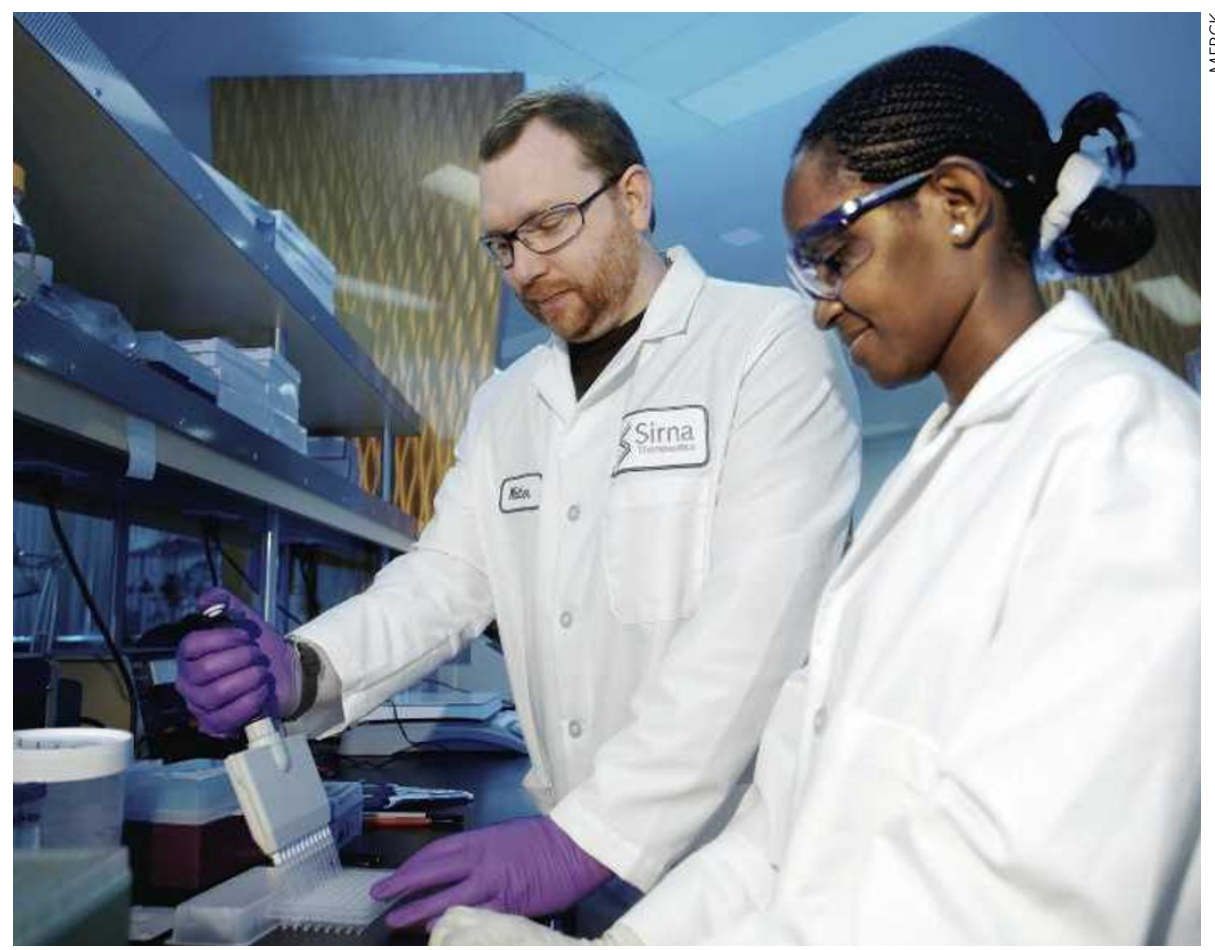

Sirna Therapeutics is developing stable siRNA compounds for silencing disease-related genes.

disrupting the viral structure. Even so, efficient and specific targeting remains an issue.

"I think that it will come down to two things: a more extensive learning of the modification of the viral structure proteins, and identification of specific target receptors so that you can have the viruses interact with cells in a very specific manner," says Verma. He points to recent work from David Baltimore's lab at the California Institute of Technology in Pasadena, in which a small monoclonal antibody was linked to a lentiviral envelope protein for targeting to specific cell types ${ }^{4}$, and to other groups trying to use small ligands and signature sequences from cell-surface receptors as promising developments for the targeting of viral vectors.

As researchers wrestle with the targeting issue, some companies have already begun to use lentiviral vectors to deliver therapeutic RNAi. Dropulic sees the ex vivo use of lentiviral transduction systems as being a critical first step. "I think using the transduced cells as the vehicles of widespread dissemination rather than thinking about direct injection of lentiviral vectors will be the way to go," he says. Lentigen is using this approach on T cells and stem cells for cancer therapies as well as for treatment of infectious diseases.

\section{The silent future}

Most experts agree that therapeutic RNAi will probably not rely on a single delivery vehicle or administration approach for all diseases. "Some diseases might require lower doses than others or could be semi-local in nature," says Robert Langer, a bioengineer at MIT, "so I think a lot may depend on the disease and treatment modality you are thinking about."

One likely beneficiary of the work on deliv- ery mechanisms are siRNA's recently uncovered siblings: microRNAs (miRNAs), which are naturally occurring siRNAs found in plants and animals. "With miRNA therapeutics, you are generally focusing on a new class of non-coding RNAs where the biology is still being discovered," says Maraganore. It is early days for this technology, but companies are now being established to work exclusively on miRNAbased therapies (see 'Thinking small').

When it comes to siRNA, Maraganore is encouraged by the early approaches to delivery being used in clinical studies. He expects the development of new delivery vehicles to continue for sometime to come. "I suspect that in 35 years scientists will continue to work on optimizing delivery approaches," he says.

And Verma thinks that the discovery of RNAi has even breathed fresh life into the still struggling field of gene therapy. "I think having RNAi technology available now gives a new impetus because it is such an effective technology — that is, if only we could deliver it."

Nathan Blow is technology editor for Nature and Nature Methods.

1. Elbashir, S. M. et al. Nature 411, 494-498 (2001).

2. Soutschek, J. et al. Nature 432, 173-178 (2004).

3. Zimmermann, T. S. et al. Nature 441, 111-114 (2006)

4. Yang L., Bailey, L., Baltimore, D. \& Wang, P. Proc. Natl Acad. Sci. USA 103, 11479-11484 (2006).

5. Medarova, Z., Pham, W., Farrar, C., Petkova, V. \& Moore, A. Nature Med. 13, 372-377 (2007).

\section{Correction}

In the Technology Feature 'The personal side of genomics' (Nature 449, 627-630; 2007) we said that Illumina uses emulsion PCR in its nextgeneration sequencing systems. In fact, the company's genome analyser uses solidphase amplification on a planar, optically transparent surface. 